\title{
Emphasizing the Nurse Practitioner Role in Addressing Unique Challenges Associated with Benzodiazepine use among the Elderly
}

NPOJ 2021, 1, pp.24-36

Jennifer Fournier MHS, PhD, NP-PHC

jfournier41@gmail.com

Robyn Gorham MN, EdD, NP-PHC ${ }^{2}$

rgorham@laurentian.ca

1. Centre for Nursing and Health Studies, Athabasca University, Athabasca, Alberta, Canada

2. School of Nursing, Laurentian University, Sudbury, Ontario, Canada

\begin{abstract}
Aim: The aim of this paper is to discuss the use of benzodiazepine medications among the elderly, and the role of a nurse practitioner within this patient population.

Background: Benzodiazepines are well known to have a negative impact on the mobility of elderly patients and to contribute to increased falls. These medications also have an impact on elderly patients' ability to perform activities of daily living and to drive. Nurse practitioners offer holistic care to elderly patients. This includes prescribing medications, monitoring therapy, deprescribing medications and identifying opportunities for safe alternatives to treat a variety of conditions.

Methods: This paper presents a narrative review focused on the effect of benzodiazepine medications on the elderly patient population, with an emphasis on the use of benzodiazepines in insomnia.

Conclusions: Findings of this review confirm the known risks associated with the use of benzodiazepines in the elderly and that these medications should be carefully considered in ongoing management. The conclusions of this review support the use of nurse practitioners in the enhancement of the healthcare of the elderly through the continuum of assessment, monitoring, deprescribing of benzodiazepine medications when appropriate, as well as opportunities to implement alternative treatments.
\end{abstract}

\section{Introduction}

Nurse Practitioners (NPs) have an important role to play in the care of elderly patients. Their holistic, evidence-based approaches, with a focus on primary care, make NPs well suited to addressing the special needs of this population (Canadian Nurses Association, 2021). The Ontario Nurse Practitioner Practice Standards are very specific with expectations for NPs who decide to prescribe controlled substances such as benzodiazepines. There is an expectation that all treatment options are considered (including pharmacological and non-pharmacological), rather than controlled substances being as first, or only choice of treatment. It is also incumbent on NPs to monitor the patient response on an ongoing basis, even if a treatment was initiated by another practitioner (College of Nurses of Ontario, 2021a). 


\section{Context}

With their interdisciplinary approaches and advanced training in health assessment, diagnosis and therapeutic management (College of Nurses of Ontario, 2021b), Canadian NPs are prepared to provide comprehensive care that is attentive to the effects of all prescribed medications, including benzodiazepines, on the wellbeing of elderly patients. Benzodiazepines are controlled substances that may be used to treat common symptoms among the elderly, such as insomnia and anxiety (Davies et al., 2018). Examples of benzodiazepines include drugs such as alprazolam (Xanax), diazepam (Valium), clonazepam (Rivotril) and lorazepam (Ativan) (Lader, 2011). Nurse practitioners are also able to provide alternatives to the use of any controlled substance, where appropriate, and to recognize opportunities to wean controlled substances where risks outweigh benefits of their use (College of Nurses of Ontario, 2021b).

\section{Approach}

This article will commence with a narrative review of the literature that summarizes what is currently known about benzodiazepines, mobility, and the older adult. The review will span a period of twenty years. Upon completion of the review, the alignment of the NP role with effective, evidence based, mobility supportive care of elderly patients using benzodiazepines, therapeutic alternatives and deprescribing will be outlined.

\section{Literature Review}

\section{Method}

A narrative review of the literature was conducted. While a narrative review includes a broad overview of a particular issue, it has the benefit of allowing the authors to use their intuition and research experience in the review (Pae, 2015). In this case, both authors have an unrestricted licensed as a Nurse Practitioners in Ontario, approximately twenty years of experience as an NP, are educated at the doctorate level, and have approximately ten years of faculty research experience. Both authors graduated at a time when the NP role was limited and clearly defined, and have worked during the transition of the role into the current full scope of practice (College of Nurses of Ontario, 2003; College of Nurses of Ontario, 2021b).

The search was limited to articles published on January $1^{\text {st }} 2000$ or later using PubMed, CINAHL, and ProQuest Nursing \& Allied Health Premium databases. Key search terms included benzodiazepine and mobility; benzodiazepine and mobility and older adult; benzodiazepine and wellbeing and older adult; benzodiazepine and functional ability and older adult. English language references were selectively retrieved. A total of 7,159 articles were initially identified for potential inclusion. An additional manual search was conducted within approved literature to retrieve other eligible articles that had been referenced or cited that warranted inclusion in this review. Final inclusion was determined on whether or not the articles fit into four general areas including; 
benzodiazepine use and risks, mobility and balance, activities of daily living (includes accidents and falls), and cognition. Prior to final inclusion in this review, article abstracts were scanned to determine appropriateness. A total of 18 articles were included for this overarching review of benzodiazepines in the elderly. The role of the Nurse Practitioner was then considered in the context of the reviewed literature.

\section{Findings}

\section{Benzodiazepine Use and Risks}

Benzodiazepines as a drug class have been identified as an independent risk factor for adverse drug effects (Gregg et al., 2016). They can impair cognition, balance and driving ability among community dwelling seniors and can increase the risk of falls (Alic et al., 2011; Gregg et al., 2016; Nana et al., 2015; National Institute of Health, 2015; Rubenstein \& Josephson, 2002). The effects of benzodiazepine medications on the elderly patient population are of particular concern given their effects on mobility and driving, and the importance of these two factors in the maintenance of autonomy and independence (Parker et al., 2002). Mobility is important for movement within one's environment and for completion of the activities of daily living (Berryman et al., 2013). Skillful management of therapies offered to elderly patients may support their mobility and contribute to enhanced safety, life satisfaction and wellbeing (Mollaoglu et al., 2010).

Guideline based criteria for prescribing benzodiazepines usually includes the presence of a relevant psychiatric diagnosis. Meanwhile, many older adults receiving benzodiazepines do not meet this criterion (Maust et al., 2012), For example, benzodiazepines are often prescribed or continued for the management of perceived insomnia. A 2015 study identified that psychotropic drugs - a class of drugs that includes benzodiazepines - were prescribed more often for older adults in Canada than they were for patients in Albania, Colombia and Brazil. Within the same study, low socioeconomic status was associated with higher frequency of psychotropic drug use, including the use of benzodiazepines (Nana et al., 2015). In a 2008 study conducted in the United States, long-term use (more than 120 consecutive days) of benzodiazepine medications was found to increase markedly with age. Among elderly patients who received benzodiazepines, $31.4 \%$ received prescriptions for long-term use. Comparatively, among patients $18-35$ years of age only $14.7 \%$ received longer-term prescriptions (Olfson et al., 2015).

\section{Mobility and Balance}

Many of the studies focused on the impact of benzodiazepine use on mobility and balance. A 2012 study investigated sedative load of benzodiazepine medications in adults 75 years of age and older (Taipale et al., 2012). The sample was random through invitation to participate. Physiotherapists assessed mobility and balance. Covariance and logistic regression were used to assess the association between sedative load and balance and mobility. Exposure to higher sedative medication load, including benzodiazepines, 
were associated with slower walking speed $(\mathrm{p}<.001)$ and prolonged Timed Up and Go Test results $(\mathrm{p}=0.005)$ (Taipale et al., 2012).

A longitudinal study focused on community-dwelling older adults followed 1000 older adults 65 years of age and older. The objective of the study was to identify the prospective association between baseline benzodiazepine use and mobility, function and pain among rural and urban dwelling older adults. Life space analysis was used to assess functioning in essential tasks of daily living and instrumental activities of daily living (Petrov et al., 2014). Benzodiazepine use was associated with greater difficulty with activities of daily living, more frequent pain and declines in mobility among rural residents. Benzodiazepine use was also found to be associated with challenges related to difficulties with activities of daily living among community-dwelling older adults and mobility decline among rural study participants (Petrov et al., 2014).

A study of mobility limitation in community dwelling seniors was undertaken by Boudreau et al. (2009) to evaluate whether benzodiazepines, opioid receptor agonists, antipsychotics, and antidepressants affected incident mobility limitation. Incident mobility limitation was defined as two self- reports of having difficulty walking a quarter mile or climbing ten steps without resting. Participants were reassessed every six months during the course of the study. Hazard analyses were conducted with adjustments for health status, demographics, health behaviours and indications for the use of medications that affect the central nervous system. By the sixth year of the study authors found that $49 \%$ of participants developed incident mobility limitations. Users of benzodiazepines, opioid receptor agonists, antipsychotics, and antidepressants showed a higher risk for incident mobility than never users (Boudreau et al., 2009).

A 2003 study assessing physical performance of community dwelling older women who use benzodiazepines assessed physical performance test results of eight hundred eighty-five women over a three-year period (Gray et al., 2003). Mobility assessment was achieved through the measurement of standing balance, walking speed (8-foot distance) and repeated chair raises. After adjustment for potential confounding variables, benzodiazepine use was associated with a greater decline in physical performance over four years than non-use. Current, long term use was related to decline while past use and recent use were not. Use of the lowest possible dosing for the shortest duration was recommended as a result of the findings (Gray et al., 2003).

A 2015 prospective cohort study sought to examine cross-sectional and longitudinal associations between benzodiazepine use and activity limitations in older adults (Carriere et al., 2015). The duration of the study was seven years. Benzodiazepine users were separated based on clinical indications into groups being treated for hypnotic, short acting anxiolytic and long acting anxiolytic purposes. Outcomes included restrictions in mobility, instrumental activities of daily living and social participation. Hypnotic benzodiazepines were associated with mobility limitations and limitations in activities of daily living. Short and long acting anxiolytics were associated with limitations in activities of daily living and with limitations in mobility. Longer acting benzodiazepines being used for anxiolytic effects were also associated with limitations in 
the performance of activities of daily living. The research team found that longer-term users of benzodiazepines were more likely to develop restrictions in all three outcomes: mobility, instrumental activities of daily living and social participation (Carriere et al., 2015).

A 2009 study assessed mobility limitations in older adult patients taking central nervous system medications. The five-year longitudinal study included 3055 participants who were mobile and functioning well at the start of the study (Boudreau et al., 2009). Dosing and mobility scores were evaluated. Mobility was measured using self-report of any difficulty walking a quarter mile or climbing ten steps without resting and these variables were measured every six months from the start of the study. In the final analysis central nervous system medication users (including those using benzodiazepines) showed higher risk for incident mobility limitation when compared to non-users at the $95 \%$ confidence interval. Findings of increased risk were also found in analysis of dose and duration response relationships (Boudreau et al., 2009).

\section{Activities of Daily Living, Accidents and Falls}

Additional studies explored more broadly to include benzodiazepines and activities of daily living, falls risk and impact on driving. A prospective cohort study examining cross sectional and longitudinal associations between benzodiazepine use and effects on activities of daily living evaluated 6600 adults over 65 years of age and followed them at 2, 4 and 7 years (Carriere et al., 2015). Mobility, instrumental participation in activities of daily living and social participation were evaluated. Chronic benzodiazepine users were found to have decreased scores on all three outcomes. Outcomes were not markedly affected by whether long or short acting benzodiazepines were used. Authors cautioned that benzodiazepine use should be limited, especially among the elderly given its effects on disability (Carriere et al., 2015).

A 2006 prospective cohort study assessed benzodiazepine use and physical disability in community dwelling older adults (Gray et al., 2006). Nine thousand ninetythree adults 65 years of age and older were enrolled. Criteria included not being disabled in mobility or activities of daily living at the start of the study. In order to be included in the study, participants had to be able to walk a half mile or climb one flight of stairs. They also had to be able to perform all of their activities of daily living independently (Gray et al., 2006). Data were collected annually over the six years of the study, over the phone for years one, two, four and five, and in person visits in years three and six. Benzodiazepine users were 1.23 times more likely to develop mobility disability than non-users. Benzodiazepine users were also 1.28 times more likely to develop deficits in the performance of activities of daily living. Finally, the authors concluded that the use of short acting benzodiazepines did not prove to have any safety benefit over the use of long acting benzodiazepines in the study population (Gray et al., 2006).

A 2017 narrative, non- systematic review of the epidemiologic research related to the use of benzodiazepines and z-drugs (Brandt \& Leong, 2017) identified that there is a converging body of evidence around the connections between benzodiazepine use and 
motor vehicle accidents, falls and fractures secondary to psychomotor impairment (Brandt \& Leong, 2017). Given the importance of movement within one's environment, especially for seniors, the connections among benzodiazepine use and driving can pose a significant safety and quality of life concern for older adults. The authors concluded that higher benzodiazepine doses, polypharmacy and the early treatment period with benzodiazepines can be particularly hazardous for patients with altered mobility in light of the drug's effects on balance and posture in older adults (Brandt \& Leong, 2017).

\section{Cognition}

A study of cognitive and physical function of older adults exposed to various categories of anticholinergic and sedative medications was undertaken (Wouters et al., 2020). Over twenty years of data was analyzed to determine the consequences of exposure to the medications. Cognitive functioning was assessed using the Mini Mental Status Examination, the Alphabet Coding Task and others. Physical functioning was assessed using the Walking Test, the Cardigan Test, The Chair Stands Test, the Balance Test and self-reported levels of functional independence. Linear mixed models were used to adjust for age, education, sex, living with a partner, BMI, depressive symptoms, comorbidities and prescribed medications. Higher drug burden was associated with poorer cognitive functioning and poorer physical functioning including longer completion time on the Cardigan Test, the Chair Stand Test, and participants demonstrated lower functional independence. The team concluded that long-term, cumulative exposure led to poorer cognitive and physical functioning among older adults (Wouters et al., 2020).

\section{Summary of Literature}

The literature related to the use of benzodiazepines in the elderly provides strong evidence of the negative impact of benzodiazepine medications on the mobility of older adults as measured through various methods across studies (Boudreau et al., 2009; Carriere et al., 2015; Gray et al., 2003; Gray et al., 2006; Petrov et al., 2014). The impact of benzodiazepine medications on ability to perform activities of daily living has been well documented (Carriere et al., 2015; Gray et al., 2006; Petrov et al., 2014) and effects on physical performance over a period of time among female patients and the general population were outlined (Gray at al., 2003; Gray et al., 2006). The effect of increasing doses on walking speed was identified (Tripale et al., 2012) and decreased social participation secondary to benzodiazepine use was identified within one study (Carriere et al., 2015).

\section{Nurse Practitioners and Benzodiazepine Medications in Elderly Populations}

\section{Medication Review/Deprescribing}

NPs are well suited to the care of elderly patients and have the ability to implement strategies that will promote the best outcomes for these patients. NPs have the knowledge and skills required to assess existing drug regimens for medications (College 
of Nurses of Ontario, 2021), like benzodiazepines, that may have an impact on the mobility of their elderly patients. Benzodiazepines are inappropriate for the treatment of insomnia in the elderly and should be avoided (Brandt \& Turner, 2014). Where patients have been prescribed benzodiazepines, the NP can identify the clinical indication the medication was intended to treat and determine whether treatment of the condition is still necessary and whether other kinds of treatments should be considered. If benzodiazepines have been inappropriately prescribed, or started in early life, or even if the therapy has been appropriately initiated, NPs are expected to apply strategies "to reduce risk of harm involving controlled substances, including medication misuse, addiction, and diversion" (College of Nurses of Ontario, 2021b, pg. 5). Therefore, the NP can assess whether there is an opportunity to deprescribe the medication.

Deprescribing of medications, particularly benzodiazepines, must be handled skillfully so as to minimize the effects of drug withdrawal (Pollman et al., 2015). Nurse practitioners engaging in deprescribing should be well versed in the process and apply evidence-based deprescribing guidelines in their approach. Using evidence-based tools to support deprescribing has been found to enhance clinician self-efficacy in a study where the sample included NPs (Farrell et al., 2018).

\section{Mobility Assessment and Monitoring}

When elderly patients are required to use benzodiazepine medications later in life for conditions other than insomnia, NPs serving as their regular healthcare providers can engage in follow up and monitor performance of mobility and activities of daily living in the clinic, via videomedicine or during home visits. Home visits to frail elderly have been shown to decrease mortality, reduce emergency department visits, decrease rehospitalizations and reduce admissions to long-term care (Coppa et al., 2018; Elkan et al., 2001). NPs are required to work collaboratively with other health care providers and community-based services (College of Nurses, 2021b). For example, during home visits, the NP can assess the home environment for safety and identify the need to bring in other members of the interdisciplinary team to incorporate mobility supports and safety equipment, such as a physiotherapist or occupational therapists. Mobility can be monitored by all team members, regardless of the drug regimen or underlying condition, to assess progress or identify deterioration and intervene accordingly. This however requires all health care providers understand the plan of care and individual accountabilities to ensure patient safety (College of Nurses, 2021b).

In the home, clinic and video visits may also benefit elderly patients by providing a source of social interaction and contact, and deficits in this area have been found among elderly patients taking benzodiazepines (Carriere et al., 2015). Where in-home and office visits are not possible or feasible, synchronous video-enabled visits can be used. Members of the interdisciplinary team may also be included. This method of ongoing assessment for elderly patients who may have transportation barriers to get into the clinic was found to be associated with patient satisfaction and timely adjustment of patient care plans (Appelman et al., 2020). 


\section{Assessment Tools}

Applying evidence-based tools is important for the monitoring and documentation of measurable clinical outcomes in NP practice. Mobility assessment for elderly patients taking benzodiazepines should include the Timed Up and Go Test to measure balance, sitting, standing and walking (Haines et al., 2007). In addition, cognitive function can be assessed using the Mini Mental Status Exam (Davey \& Jamieson, 2004) and the Montreal Cognitive Assessment tool (Nasreddine et al., 2005). The NP would be responsible for accurate monitoring and documentation of these parameters at regular intervals, which would facilitate recognition of any deterioration from potential continued use of benzodiazepines, and highlight the need for changes in the plan of care (College of Nurses, 2021b).

\section{Non-Pharmacologic Management}

Sleep related complaints are very common among the elderly population (Patel et al., 2018). As many as $50 \%$ of older adults present to clinicians with some kind of sleep related concern (Patel et al., 2018). Some patients who take benzodiazepines were originally prescribed them in order to facilitate sleep. Meanwhile, current guidelines discourage this practice (Brandt \& Turner, 2014). NPs can incorporate comprehensive assessment of sleep related complaints and determine whether further investigations are warranted. Going forward, NPs can introduce cognitive behavioural therapy (CBT-I) to assist their patients with sleep related complaints such as sleep latency and insomnia. CBT-I combines patient training in stimulus control, relaxation, and additional strategies in a comprehensive program to address distorted thinking about sleep and to correct sleep inhibiting behaviours. A full CBT-I program can lead to improvement in $70-80 \%$ of participants (Pigeon, 2010).

Nurse practitioners can also conduct and encourage participation in falls prevention programs for elderly patients taking benzodiazepines. Given their nursing foundations, NPs have relevant training in the facilitation of group sessions, adult teaching, learning and coaching and the physical changes of aging. Falls prevention programs have been well supported in the literature (McInnes \& Askie, 2004). In addition, group falls prevention programs have been identified as a good source of social interaction for their participants (McInnes \& Askie, 2004).

\section{Conclusion}

Nurse practitioners caring for elderly patients receiving benzodiazepine medications are in a unique position to monitor benzodiazepine use and mobility given their ongoing relationships with their patients. The effectiveness of in clinic, home visit and video assessment have been identified. The use of evidence-based assessment tools for monitoring and the implementation of falls prevention programs are supported by the literature and show promise. The importance of NP deprescribing has also been outlined. It is important for primary healthcare professionals, such as NPs to evaluate the medication profiles of elderly patients with attention to benzodiazepine type medications 
given potential negative effects. The limited appropriate role of benzodiazepine use in the elderly population should lead to more widespread use of non-drug approaches such as CBT-I. This cost effective, accessible and effective program can be delivered by NPs to individual patients and groups of patients in clinic, in home and over video systems. NPs working in family practice should consider participating in training that prepares them for skilled delivery of CBT-I in their practices.

Although non-pharmacological therapy should be used to its full potential where appropriate, NPs remain responsible for monitoring how a patient responds to pharmacological therapy, and to adjust (even discontinue) the medication based on patient response on an ongoing basis (College of Nurses of Ontario, 2021b). NPs have education in deprescribing controlled substances and can perform this function where the treatment is no longer required, or a change in dose is required. Where safer, more suitable treatments exist, they can wean treatments independently or in conjunction with other allied health professionals. For example, community pharmacists are well suited to support patient care in their ability to identify and signal to prescribers any inappropriate medication use (Urru et al., 2015).

In addition to carefully managing medications, NPs possess a variety of skills that facilitate the introduction of safe, mobility supportive approaches to the clinical management of a variety of health conditions affecting elderly patients. These may include group education for chronic symptom management, cognitive behvioural therapy for mental health challenges and leading falls prevention programs. Some NPs offer cognitive behavioural therapy specially designed for insomnia (CBT-I). This approach has proven to be an effective alternative to benzodiazepines and other similar medications used in the management of insomnia in the elderly (Cassidy-Eagle et al., 2017). CBT-I has been shown to lead to significant improvement in sleep and in executive functioning (Cassidy-Eage et al., 2017), and in significantly reduced pain ratings among elderly patients with osteoarthritis (Vitiello et al., 2009).

This paper offered a review of the risks related to benzodiazepine use in the elderly and the potential benefits that can be gained through NP care. Given their holistic approaches and broad skill base, NPs are well suited to care for elderly patients, and specifically those requiring ongoing monitoring, deprescribing and non-pharmacologic approaches to the management of clinical concerns. With their education in pharmacology, coaching, teaching, community care and group leadership, NPs are well positioned to effectively deliver the evidence-based and person-centered care required to manage conditions such as insomnia, impaired mobility and falls risk among our most vulnerable citizens.

\section{References}

Alic, A. Pranjic, N., \& Ramic, E. (2011). Polypharmacy and decreased cognitive abilities in elderly patients. Med Arch, 65(2), 102-105. PMID:21585185. 
Appelman, E., O’Connor, M., Rockefeller W., Morin, P. \& Moo, L. (2020). Using video telehealth to deliver patient - centered collaborative care: The g-impact pilot. Clinical Gerontologist, 1-10. DOI: 10.1080/07317115.2020.1738000

Berryman, N., Bherer, L., Nadeau, S., Lauziere, S., Lehr, L., Bobeuf, F., Kergoat, M., Vu, T \& Bousquet, L. (2013). Executive functions, physical fitness and mobility in well-functioning older adults. Experimental Gerontology, 48, 1402-1409. DOI: 10.1016/j.exger.2013.08.017

Boudreau, R., Hanlon, J., Roumani, Y., Studenski, S., Ruby, C., Wright, R., Hilmer, S., Shorr, R., Bauer, D., Simonsick, E., \& Newman, A. (2009). Central nervous system medication use and incident mobility limitation in community elders: the health, aging, and body composition study. Pharmacoepidemiology \& Drug Safety, 18(10), 916-922. DOI: http://doi.org/10.1002/pds.1797.

Brandt, J. \& Leong, C. (2017). Benzodiazepines and z-drugs: An updated review of major adverse outcomes reported in epidemiologic research. Drugs Research and Development, 17(4), 493-507. doi: 10.1007/s40268-017-0207-7

Brandt, N. \& Turner, T. (2014). Potentially inappropriate medications in older adults: A review of the 2012 Beers Criteria and the implications in persons with dementia. Mental Health Clinician, 4(4), 166-169. https://doi.org/10.9740/mhc.n204331

Canadian Nurses Association. (2021). Nurse Practitioner. Retrieved online: https://cnaaiic.ca/en/nursing-practice/the-practice-of-nursing/advanced-nursingpractice/nurse-practitioners

Carriere, I., Mura, T., Peres, K, Norton, J., Jaussent, I., Edjolo, A., Rouaud, O., Berr, C., Ritchie, K., \& Ancelin. M. (2015). Elderly benzodiazepine users at increased risk of activity limitations: Influence of chronicity, indications, and duration of action The three-city cohort. American Journal of Geriatric Psychiatry, 23(8), 1545-7214. DOI: org/10.1016/j.jagp.2014.10.006

Cassidy-Eagle, E., Sibern, A., Unti. L., Glassman, J. \& O’Hara, R. (2017).

Neuropsychological functioning in older adults with mild cognitive impairment and insomnia randomized to CBT-I or Control Group. Clinical Gerontology, 41(2). 136-144. DOI: org/10.1080/07317115.2017.1384777

College of Nurses of Ontario (2003). Standards of practice for registered nurses in the extended class (Revised 2003).

College of Nurses of Ontario (2021a). Extended Class. Retrieved online June 24, 2021 from https://www.cno.org/en/become-a-nurse/classes-of-registration/extendedclass/ 
College of Nurses of Ontario (2021b). Practice Standard, Nurse Practitioner. Retrieved online June 24, 2021 from https://www.cno.org/globalassets/docs/prac/41038_strdrnec.pdf

Coppa, D., Winchester, S., \& Barcelos, M. (2018). Home-based nurse practitioners demonstrate reductions in rehospitalizations and emergency department visits in a clinically complex patient population through an academic-clinical partnership. Journal of the American Association of Nurse Practitioners, 30(6), 335-343. DOI: $10.1097 / J X X .0000000000000060$

Davey, R., \& Jamieson, S. (2004). The validity of using the mini mental state examination in NICE dementia guidelines. Journal of Neurology and Neurosurgery Psychiatry, 75: 343-344. PMID: 14742628

Davies, S., Jacob, B., Rudoler, D., Zaheer, J., de Oliveira, C. \& Kurdyak, P. (2018). Benzodiazepine prescription in Ontario residents aged 65 and over: a populationbased study from 1998 to 2013. Therapeutics advances in psychopharmacology, 8(3), 99-114. doi: $10.1177 / 2045125317743651$

Elkan, R., Kendrick, D., Dewey, M., Hewitt, J., Blair, M., Williams, D. \& Brummel, K. (2001). Effectiveness of home based support for older people: systematic review and meta-analysis. British Medical Journal, 323, 719-25.

DOI: $10.1136 / \mathrm{bmj} .323 .7315 .719$.

Farrell, B., Richardson, L., Raman-Wilms, L., de Launay, D., Alsabbagh, M. \& Conklin, J. (2018). Self-efficacy for deprescribing: A survey for health care professionals using evidence-based deprescribing guidelines. Research in Social and

Administrative Pharmacy, 14(1), 18-25. DOI: 10.1016/j.sapharm.2017.01.003.

Gray, S., Penninx, B., Blough, D., Artz, M., Guralnik, J., Wallace, R., Buchner, D., and LaCroix, A. (2003). Benzodiazepine use and physical performance in communitydwelling older women. Journal of the American Geriatrics Society, 51(11), 1563 70. DOI: $10.1046 / \mathrm{j} .1532-5415.2003 .51502 . x$

Gray, S., LaCroix, A., Hanlon, J., Penninx, B., Blough, D., Leveille, S., Artz, M., Guralnik, J., \& Buchner, D. (2006). Benzodiazepine use and physical disability in community-dwelling older adults. Journal of the American Geriatrics Society, 54(2), 224-230. DOI: 10.1111/j.1532-5415.2005.00571.x

Gregg, J., Tyson, R. \& Cook. D. (2016). Benzodiazepines and geriatrics: What clinical practice strategies can be used to reduce the inappropriate prescribing? Rehabilitation Nursing, 41(5), 270-275. DOI: 10.1002/rnj.288

Haines, T, Kuys, S., Morrison, G., Clarke, J., Bew, P., \& McPhail, S. (2007). Development and validation of the balance outcomes measure for elder 
rehabilitation. Archives of physical medical rehabilitation, 88(12), 1614-1621. DOI: org/10.1016/j.apmr.2007.09.012

Lader, M. (2011). Benzodiazepines revisited- will we ever learn? Addiction, 106(12), 2086-2109. DOI: $10.1111 / j .1360-0443.2011 .03563 . x$

Maust, D., Mavandadi, S., Benson, A., Streim, J., DiFilippi, S., Snedden, T. \& Oslin, D. (2012). Telephone-based care management for older adults initiated on psychotropic medication. International Journal of Geriatric Psychiatry, 28(4), 410416. DOI: $10.1002 / \mathrm{gps} .3839$

McInnes, E. \& Askie, L. (2004). Evidence review on older people's views and experiences of falls prevention strategies. Worldviews on Evidence Based Nursing, 1(1), 20-37. DOI: $10.1111 / \mathrm{j} .1741-6787.2004 .04013 . x$.

Mollaoglu, M., Tuncay, F., \& Fertelli, T. (2010). Mobility disability and life satisfaction in elderly people. Archives of Gerontology and Geriatrics, 51(3), 115-119. DOI: org/10.1016/j.archger.2010.02.013

Nana, G., Doulougou, B., Gomez, F., Ylli, A., Guralnik, J \& Zunzunegui, M. (2015). Social differences associated with the use of psychotropic drugs among men and women aged 65 to 74 years living in the community: the international mobility in aging study (IMIAS). BMC Geriatrics, 15(85), 1-10. DOI: 10.1186/s12877-0150083-3

Nasreddine, Z., Phillips, N., Bedirian, V., Charbonneau. S., Whitehead, V., Collin, I., Cummings, J. \& Chertkow, H (2005). The Montreal cognitive assessment, MOCA: a brief screening tool for mild cognitive impairment. Journal of the American Geriatric Society, 53(4), 695-699. DOI: 10.1111/j.1532-5415.2005.53221.x.

National Institute of Health. (2015). Benzodiazepine often used on older people despite risks. Retrieved online July 9th 2021 from https://www.nih.gov/news-events/nihresearch-matters/benzodiazepine-often-used-older-people-despite-risks

Olfson, M., King, M. \& Schoenbaum, M. (2015). Benzodiazepine use in the United States. JAMA Psychiatry, 72(2) 136-42. DOI:10.1001/jamapsychiatry.2014.1763.

Pae, C. (2015). Why systematic review rather than narrative review? Psychiatry Investigation, 12(3), 417-419. doi: 10.4306/pi.2015.12.3.417

Parker, M., Baker, P., and Allman, R. (2002). A life-space approach to functional assessment of mobility in the elderly. Journal of Gerontological Social Work, 35(4), 35-55. DOI org/10.1300/J083v35n04_04

Patel, D., Steinberg, J. \& Patel, P. (2018). Insomnia in the elderly: a review. Journal of Clinical Sleep Medicine, 14(6), 1017-1024. DOI: 10.5664/jcsm.7172 
Petrov, M., Sawyer, P., Kennedy, R., Bradley, L., Aliman, R. (2014). Benzodiazepine (BZD) use in community-dwelling older adults: Longitudinal associations with mobility, functioning and pain. Archives of Gerontology \& Geriatrics, 59(2), 331-7. DOI org/10.1016/j.archger.2014.04.017

Pigeon, W. (2010). Treatment of adult insomnia with cognitive-behavioural therapy. Journal of Clinical Psychology, 66(11), 177-182. DOI: org/10.1002/jclp.20737

Pollman, A., Murphy, A., Bergman, J., and Gardner, D. (2015). Deprescribing benzodiazepines and Z-drugs in community-dwelling adults: a scoping review. BMC Pharmacology and Toxicology, 16(19), 1-12. DOI: 10.1186/s40360-0150019-8.

Rubenstein, L., \& Josephson, K. (2002). Risk factors for falls: A central role in prevention. Generations: Journal of the American Society on Aging, 26(4), 15-21. Retrieved June 24, 2021 from https://www.jstor.org/stable/26555172

Taipale, H., Bell, J., Gnjidic, D., Sulkava, R. \& Hartikainen, S. (2012). Sedative load among community-dwelling people aged 75 years or older: Association with balance and mobility. Journal of Clinical Psychopharmacology, 32 (2), 218-244. DOI: $10.1097 / \mathrm{JCP} .0 \mathrm{~b} 013 \mathrm{e} 3182485802$.

Urru, S., Pasina, L., Minghetti, P. \& Giua, C. (2015). Role of Community pharmacists in the detection of potentially inappropriate benzodiazepines prescriptions for insomnia. International Journal of Clinical Pharmacy, 37(6), 1004-108. http://dx.doi.org.librweb.laurentian.ca/10.1007/s11096-015-0166-4

Vitiello, M, Bybarczyk, B., Von Korff, M., \& Stepanski, E. (2009). Cognitive behavioural therapy for insomnia improves sleep and decreases pain in older adults with co-morbid insomnia and osteoarthritis. Journal of Clinical Sleep Medicine, 5(4), 355-362. DOI: org/10.5664/jcsm.27547

Wouters, H., Hilmer, S., Gnjidic, D., Campen, J., Teichert, M., Van Der Meer, H., Schaap, L., Huisman, M., Comjis, H., Denig, P., Lamoth, D., \& Taxis, K. (2020). Long-term exposure to anticholinergic and sedative medications and cognitive and physical function in later life. The Gerontological Society of America, 75(2), 357365. DOI: 10.1093 /gerona/glz019 